\title{
A Fault Tolerance Optimization Model of the China Railway Geographic Network Topological Structure
}

\author{
Fenling Feng, ${ }^{1}$ Ziwen Tang, ${ }^{1}$ and Lei Wang ${ }^{1,2}$ \\ ${ }^{1}$ School of Traffic and Transportation Engineering, Central South University, Changsha 410075, China \\ ${ }^{2}$ Wuxi Railway Station, Shanghai Railway Bureau, Wuxi 214005, China \\ Correspondence should be addressed to Fenling Feng; ffl0731@163.com
}

Received 17 July 2014; Revised 2 October 2014; Accepted 2 October 2014

Academic Editor: Wuhong Wang

Copyright (c) 2015 Fenling Feng et al. This is an open access article distributed under the Creative Commons Attribution License, which permits unrestricted use, distribution, and reproduction in any medium, provided the original work is properly cited.

\begin{abstract}
With degree distribution characteristics of the China Railway geographic network, an optimization model from macro to micro is established on the relative entropy. Firstly, Poisson distribution of the China Railway geographic network is verified and fitted. Secondly, the "Twelfth Five-Year Plan" of the railway geographic network is chosen as an example on which a macro model is built. Finally, the "Twelfth Five-Year Plan" of Guangzhou Railway Group's geographic network is chosen as the other example on which a micro model is built and our optimization scheme is proposed. Results reveal that, for improving the railway network fault tolerance, from the macroscopic aspect the "Twelfth Five-Year Plan" railway network should strengthen the railway agglomeration degree; then the microscopic optimization model is able to improve the fault tolerance effectively.
\end{abstract}

\section{Introduction}

Since the first railway was built in 1865 , China Railway has kept developing. After the implementation of reform and opening up policy in 1980s, great progress was made on the railway network. On October 26, 2012, a huge and complex railway network was established. The Ministry of Railway of P.R.C (the (former) Ministry of Railways of China was dissolved in accordance with the decision by The 12th National People's Congress in March 2013. Of its duties, development plan, safety, regulation, and inspection were taken up by State Railway Administration, a new department administrated by the Ministry of Transport of P.R.C. Construction and management were taken up by China Railway Corporation) website published "The 12th Five-year Railway Network Plan" and the "National Rapid Rail Network Plan," both of which described the development direction of China Railway in the next five years. A railway network should be analyzed on its overall performance and its plan should be evaluated from an external index. The research on the railway network topology structure with statistical methods regards the railway network as a complicated system, which will support planning, constructing, and implementing the network with theoretical foundations. The fault tolerance research on complex network topology structure is an important and essential aspect in the field of complex system study. Therefore, the research on fault tolerance of the railway topology network is critical.

Many scholars researched the fault tolerance of complex networks. Albert et al. [1] first studied the robustness of random networks and scale-free networks under random failures and deliberate attacks. Broder et al. [2] discussed the robustness of Internet networks under random failures and deliberate attacks by researching on WWW networks. Cohen et al. [3, 4] discussed the robustness of Internet networks under random failures and malicious attacks based on percolation theory. Feng et al. [5] studied connection robustness and recovery robustness based on the connectivity and recovery ability of the network. Moreover, many scholars carried out researches on other networks, such as China's railway interchange network [6], public transport networks [7], and complex military networks [8].

As for the optimization of fault tolerance in a complex network, Shargel et al. [9] improved the resistance ability of 
TABLE 1: Degree distribution of the China Railway network.

\begin{tabular}{lcccccccccc}
\hline Degree & 1 & 2 & 3 & 4 & 5 & 6 & 7 & 8 & 9 & 10 \\
\hline $\begin{array}{l}\text { Degree proportion of railway } \\
\text { network in 2008 }\end{array}$ & 0.2077 & 0.2524 & 0.2204 & 0.1725 & 0.0895 & 0.0383 & 0.0096 & 0.0064 & 0.0032 & - \\
\hline $\begin{array}{l}\text { Degree proportion of railway } \\
\text { network in the "Twelfth }\end{array}$ & 0.1024 & 0.1667 & 0.2571 & 0.2262 & 0.1429 & 0.0405 & 0.0238 & 0.0214 & 0.0119 & 0.0071 \\
\begin{tabular}{l} 
Five-Year Plan" \\
\hline
\end{tabular}
\end{tabular}

the BA model by adjusting its parameters which are identified for networks' better resistance ability. Paul et al. [10] made an analysis on the optimization of scale-free networks by comparing the advancement in double power law networks and bimodal distribution networks, and so forth, in order to improve the resistance ability against random failures and deliberate attacks at the same time. In terms of the means of attacks, Tanizawa et al. [11] demonstrated the node degree distribution of networks with better resistance ability when random failures and selective attacks arise regularly. Wang and $\mathrm{He}[12,13]$ stated that entropy optimization can improve the network fault tolerance. By changing the topology structure, the network fault tolerance capacity will be improved. They illustrated the feature that the stronger the fault tolerance, the less the hubs and the lower the node degree. However, with enhanced network fault tolerance and better transmission efficiency, the network synchronous ability seems to be weakened.

Studies reviewed covering the complex network fault tolerance optimization mostly focus on adjusting one or few microscopical parameters to improve the tolerance. This paper proposes a new tolerance optimization method using a set from macro to micro fault tolerance, proposing to utilize the optimal value of the overall network fault tolerance as the degree distribution is macroscopic. Then the method would be used from the proposed microscopic local network fault tolerance optimization to the local specific network optimization.

\section{Poisson Distribution of China's Railway Network}

According to researches by He Cheng [13], Zhao et al. [14] and other scholars, the degree distribution of China's Railway geographic network is similar to a tree structure aligning with Poisson distribution. To prove that the network structure is Poisson distributed, we select the railway network in the year of 2008 and in the "Twelfth Five-Year Plan" and the second and the third nodes' degree weighs more in the degree distribution of the China which agrees with characteristics of the Poisson distribution; see Table 1 and Figure 1.

Fitting with Poisson distribution function, the function of degree distribution of the 2008 China Railway geography network is $P(x=k)=\left(2.2882^{k} / k !\right) e^{-2.2882}$.

And the function of the degree distribution of the “Twelfth Five-Year Plan” is $P(x=k)=\left(3.5853^{k} / k !\right) e^{-3.5853}$.
For the comparison of China Railway geography network degree distribution, see Figure 2.

\section{Fault Tolerance Macroscopic Optimization Model}

Cohen et al. (2000) stated that the stronger the network heterogeneity, the stronger the fault tolerance. Traditionally, from the perspective of information theory, information entropy is commonly used as a measure of the network heterogeneity.

Relative entropy measures the distance between two random distributions. In statistics, it corresponds to the logarithm expectation of likelihood ratios. The relative entropy or Kullback-Leibler distance of two probability density functions $p(x)$ and $q(x)$ is defined as follows:

$$
D\left(\frac{p}{q}\right)=\sum_{x \in A} p(x) \log \frac{p(x)}{q(x)} .
$$

Obviously, uniform network has the worst heterogeneity. Therefore, this study uses the relative information entropy based on the real network and takes the regular network degree distribution as the measurement, which is also adopted to research on fault tolerance. The relative entropy measurement is more representative revealing more comparability than traditional information entropy ones.

All the nodes in the homogeneous network are evenly distributed. The proportion of every node degree is $1 / n$, which is for any node degree $x_{i} \in A, i=1,2, \ldots, n, p\left(x_{i}\right)=$ $1 / n$ so the information entropy of the uniform network is

$$
H_{\text {max }}=-\sum_{i=1}^{n} \frac{1}{n} \log _{2} \frac{1}{n}=-n \frac{1}{n} \log _{2} \frac{1}{n}=\log _{2} n .
$$

We set the node degree distribution density function of the railway network as $p(x)$, so according to the relative entropy formula, fault tolerance measurement $C$ is

$$
\begin{aligned}
C & =D(p \| q) \\
& =\sum_{x \in A} p(x) \log \frac{p(x)}{q(x)} \\
& =\sum_{x \in A} p(x) \log (n p(x)) .
\end{aligned}
$$




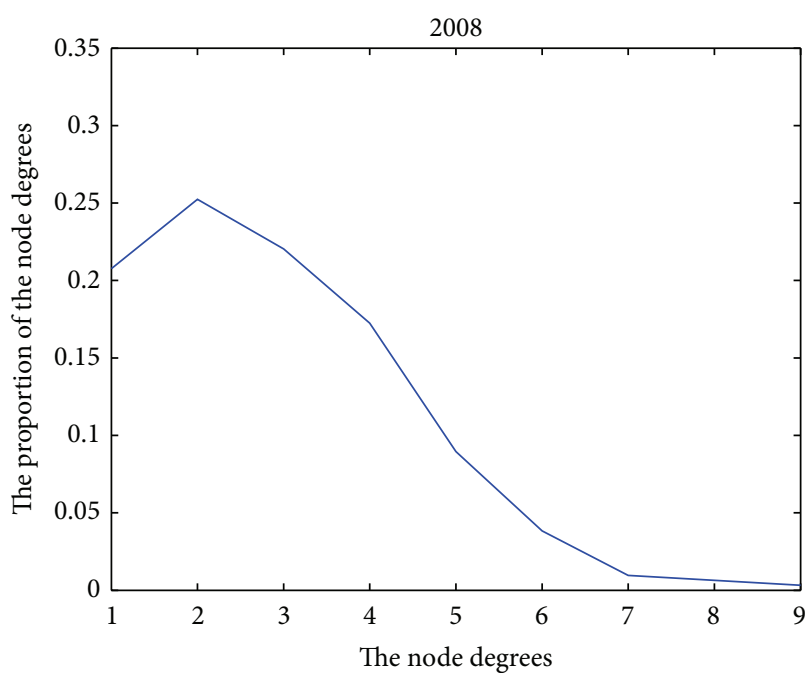

(a)

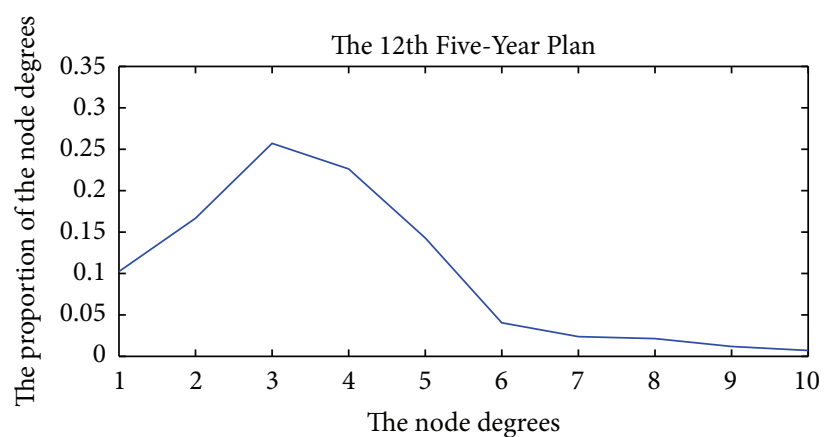

(b)

FIGURE 1: Degree distribution of the China Railway geography network.

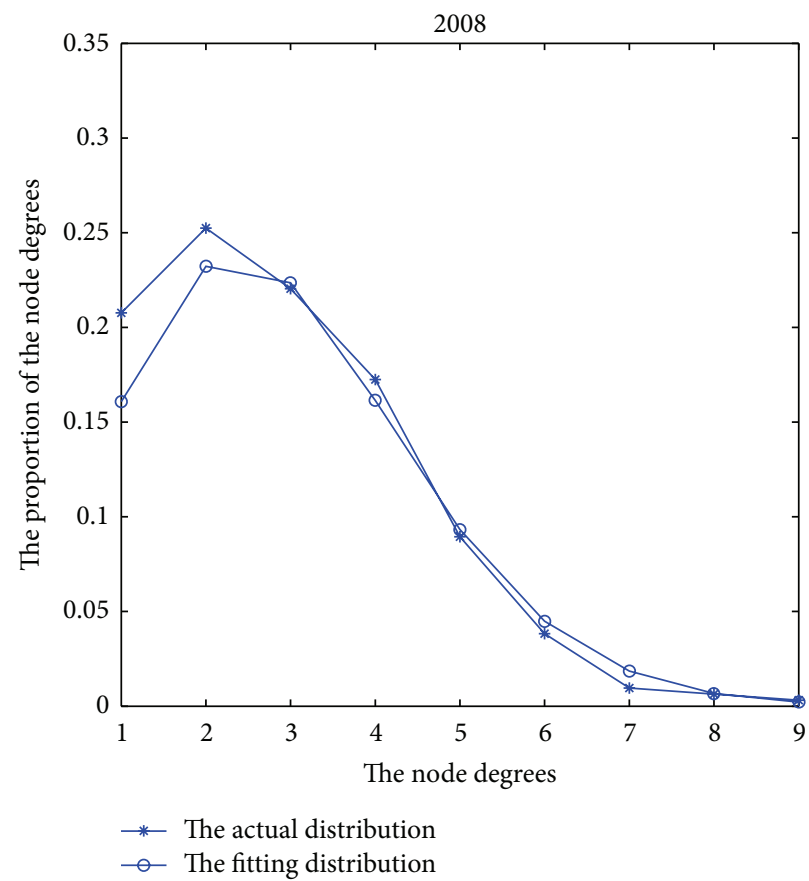

(a)

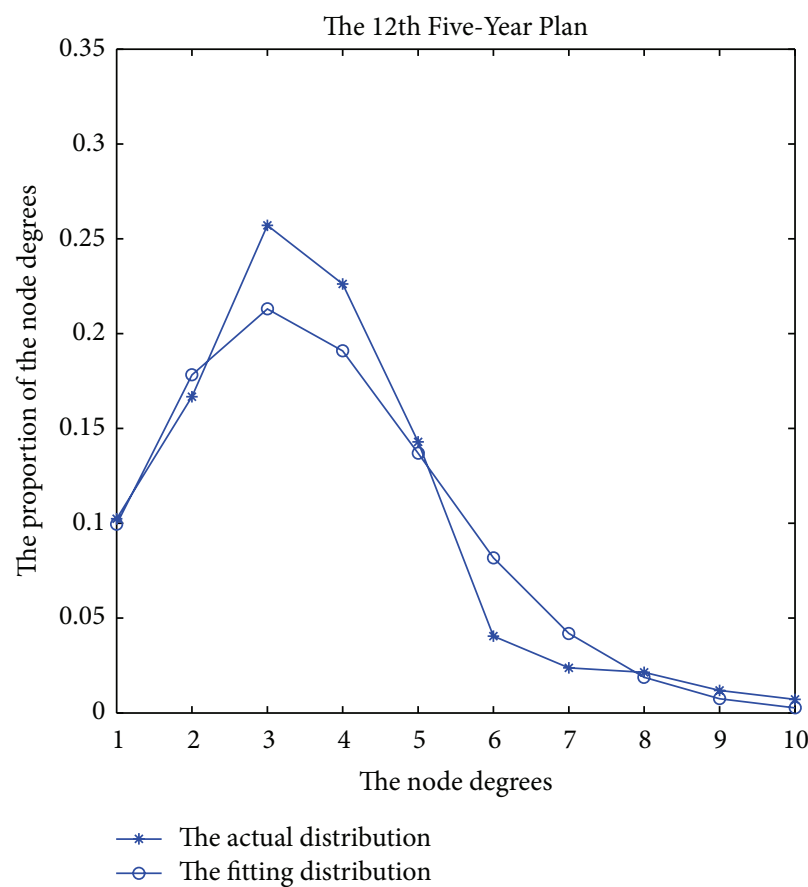

(b)

FIgURE 2: Comparison between actual degree distribution and fitting degree distribution of China Railway geography network.

Based on the above discussion, the fault tolerance macroscopic optimization model is established, the object of which is the "Twelfth Five-Year Plan" of the China Railway geography network.

3.1. The Basic Model. Due to the fact that the optimum of the relative entropy is considered as the optimal fault tolerance, the study uses the relative entropy as the objective function to find the fault tolerance optimization by adjusting the scale in the network under the condition that keeps the cost, efficiency, and other actual factors and constraints constant. Based on all the assumptions, the basic model is as follows:

$\max H$ (Relative entropy)

s.t. Cost constraints

Efficiency constraints

Practical constraints. 


\subsection{Model Implementations}

(1) The Objective Function. Because the degree distribution of China railway geographic network fits in the Poisson distribution, we can determine the relative entropy function, which is

$$
\begin{gathered}
H=\sum_{k \in A} p(k) \log (n p(k)), \\
H=\sum_{k \in A} \frac{\lambda^{k}}{k !} e^{-\lambda} \log \left(n \frac{\lambda^{k}}{k !} e^{-\lambda}\right) .
\end{gathered}
$$
is

The objective function for the maximum relative entropy

$$
\max H=\max \sum_{k \in A} \frac{\lambda^{k}}{k !} e^{-\lambda} \log \left(n \frac{\lambda^{k}}{k !} e^{-\lambda}\right)
$$

(2) Cost Constraints. Limited by the budgets, railway construction expansion scale is constrained which reflects on the model as the following formula indicates, whereas the total number of nodes is $n$, which is a constant, and the planned scale is $n_{0}$. Therefore, cost constraints are

$$
n<a n_{0} \quad(a>1)
$$

(3) Constraints in Efficiency. The efficiency of the railway network is mainly determined by the average distance. Because the degree distribution of China Railway geography network is Poisson distributed, we can conclude that the network is random. A large number of researches state that the average shortest distance and the logarithm of scale are positive correlated, which means $n<a n_{0}(a>1)$. When the average distance is constant, cost constraints equal efficiency constraints.

(4) The Average Node Degree Distribution Constraints. According to the current circumstances, the main node degree in the geographical network is 2 or 3 . This paper emphasizes mainly hubs and ignores the secondary railway stations; then the node degree for most of the selected sites is 3 or 4 . As for those whose node degree is larger than 4 , the node degree size is negatively correlated with the proportion. Therefore, the average node degree falls into the scope of (3, 4 ), which also determines the overall structure of the railway. In order to keep the railway structure constant, we set the average node degree as

$$
3<\lambda<4
$$

(5) Planning Strategy Constraints. The "Twelfth Five-Year Plan" focuses on developing Western China and the key for the construction plan is to build new railway sites as well as extension of these sites. We will ensure that the proportion of these sites does not decrease. Therefore, we set $s$ as the proportion of the sites whose node degree is less than or equal to 3 and the strategic constraints are shown in the following equation:

$$
\begin{gathered}
\sum_{k \in\{1,2,3\}} p(k)>s, \\
\text { Namely, } \sum_{k \in\{1,2,3\}} \frac{\lambda^{k}}{k !} e^{-\lambda}>s .
\end{gathered}
$$

In conclusion, assuming the maximum node degree remains the same, the macro optimization model for fault tolerance is as follows:

$$
\begin{array}{ll}
\max & H(\lambda, n)=\sum_{k \in A} \frac{\lambda^{k}}{k !} e^{-\lambda} \log \left(n \frac{\lambda^{k}}{k !} e^{-\lambda}\right) \\
\text { s.t. } \quad & n<a n_{0} \\
& 3<\lambda<4 \\
& \sum_{k \in\{1,2,3\}} \frac{\lambda^{k}}{k !} e^{-\lambda}>s .
\end{array}
$$

3.3. The Model Results. Under conditions that cost efficiency keeps fixed, railway structure remains the same, strategic constrains stay unchanged, and fault tolerance in the "Twelfth Five-Year Plan" degree gets maximized, the average node degree is calculated to be 3.7464 , which is improved compared with the original value of 3.5853 . The improvement requires that new railway lines, if possible, connect with the existing stations and lines between new sites should be increased.

\section{Fault Tolerance Microscopic Optimization Model}

This paper establishes a microscopic optimization model of fault tolerance in which the Guangzhou Railway Group railway network is the object. It targets finding the maximum relative entropy by keeping cost constrains and efficiency constrains constant while maximizing node degree achieving the macroscopic optimization. And the basic model is as follows:

$$
\max H \text { (Relative entropy) }
$$$$
\text { s.t. Cost constraints }
$$$$
\text { Degree constraints }
$$

Practical constraints.

4.1. Algorithm Ideas. According to the basic model, determine the algorithm.

Step 1 (initialization). According to the real network diagram, geographically adjacent map and distance map are constructed. The "Twelfth Five-Year Plan" is regarded as the initial policy sets. The number of iterations of the network based on the scale of the networks is determined. 
(1)
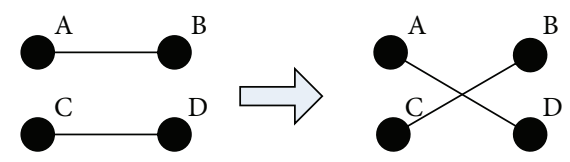

(2)

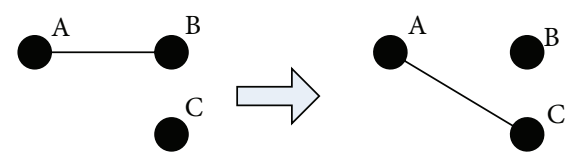

FIGURE 3: Exchanging lines (1) and changing lines (2).
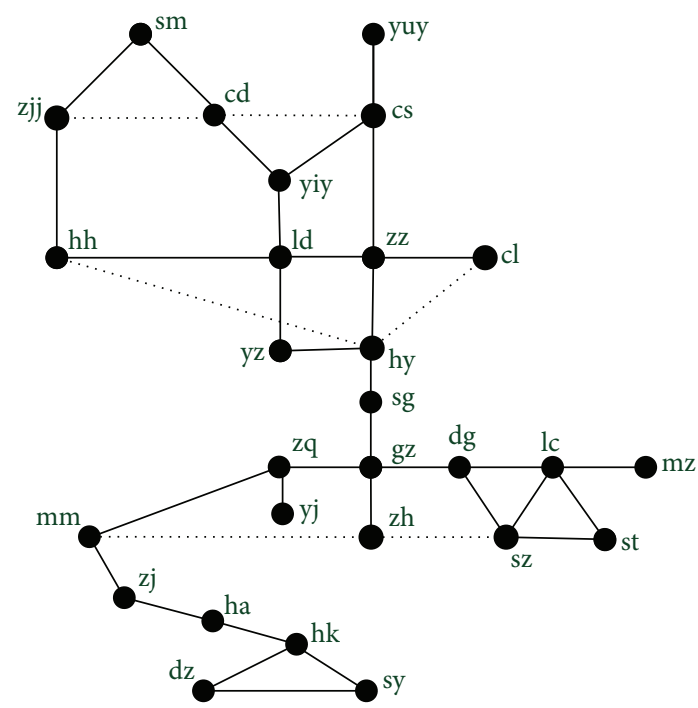

_ Existing lines

Planning lines

Figure 4: The "Twelfth Five-Year Plan" of railway geographic network diagram of Guangzhou Railway Bureau.

Step 2 (construction of strategy candidate groups). Select one pair of lines from the planned routes, exchange or change them as Figure 3 suggests, and iterate all the situations. The strategy candidate groups are established.

Step 3 (finding the optimal efficiency). The average distance reflects the distance attribute in the network, which represents the efficiency of the network. The optimized network requires better efficiency than the original.

Step 4 (seeking the optimal costs). In order to reduce the cost, the actual distance increase after the optimization should be within a certain limit.

Step 5 (macroscopic optimal screening). In addition to considering the fault tolerance of the regional railway bureau, we should also improve the national rail network fault tolerance. Therefore, it is necessary to make the optimized node degree close to the average node degree in the macroscopic optimization.
Step 6 (choosing the optimal fault tolerance). After all the work above, select the optimal strategy with the maximum relative entropy from the remaining cluster.

Step 7. The optimal strategy is exchanged to replace the initial adjacency matrix and to generate a new policy set. If the number of iterations is less than the measured value, then restart from Step 2. If it is greater than the number of iterations, the final new strategy sets should be evaluated by the level of efficiency and cost evaluation, taking the optimal strategy as the final policy set.

4.2. Guangzhou Railway Bureau Railway Network in the "Twelfth Five-Year Plan": An Example. Guangzhou Railway Bureau railway network in the "Twelfth Five-Year Plan" contains 28 nodes, 39 edges, which contain 33 existing lines and six planned lines (Figure 4).

Assume the planned network average distance is $l_{0}$, the actual distance is $L_{0}$, the average degree is $l$, the average distance of optimized network is $l$, the actual distance is $L$, and the average degree is $k$; then take constraints magnitudes $a_{1}, a_{2}, a_{3}$ as the rise of the optimized network relative to the original planning network. There are constraints:

$$
\begin{gathered}
\frac{\left(l-l_{0}\right)}{l_{0}}<a_{1}, \\
\frac{\left(L-L_{0}\right)}{L_{0}}<a_{2}, \\
\left|\frac{\left(k-k_{0}\right)}{k_{0}}\right|<a_{3} .
\end{gathered}
$$

It means that both the average distances and the actual distance have upper limit after optimization, and the average degree is controlled in a certain range. Based on the actual distance adjacency matrix, the average distance is 7.514 calculated from local railway in geographic network. For the distance of those pairs of sites is greater than $400 \mathrm{~km}$, it is estimated to be 400 kilometers. And the actual distance is $12,707.71 \mathrm{~km}$, with an average degree of 3.7464 . Constraints are assumed a 5\% margin:

$$
\begin{gathered}
\frac{(l-7.514)}{7.514}<5 \%, \\
\frac{(L-12707.71)}{12707.71}<5 \%, \\
\left|\frac{(k-3.7464)}{3.7464}\right|<5 \% .
\end{gathered}
$$

After calculation, the initial relative entropy for local railway network is 0.5375 . Obtained by the local optimization model, the optimal relative entropy converges to 0.5392 , indicating that the local optimization model can improve network fault tolerance. This optimal fault tolerance indicates that a candidate cluster network, due to the impact of the actual terrain, may be difficult to achieve optimization of the new line. Therefore it is required to be selected based on cost and efficiency indicators. 


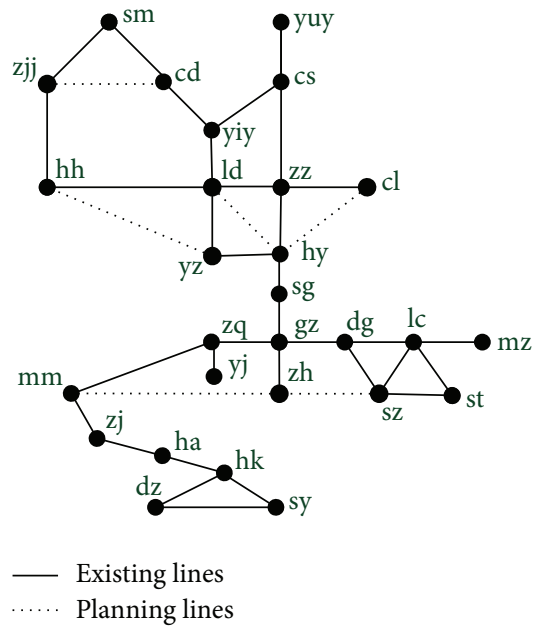

(a)

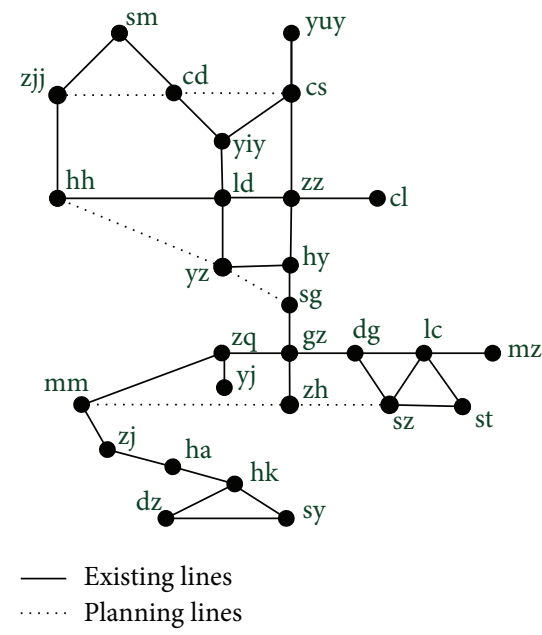

(b)

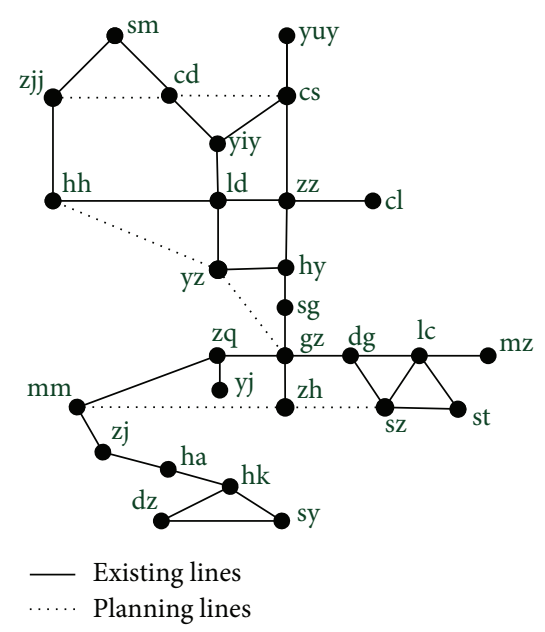

(c)

FIgURE 5: Local railway network optimization geographic figure.

We calculate the 18 candidate clusters. Then we find out the cost and the efficiency are increased compared with the original planning of the network. The average distance increases compared with the actual distance. It may reduce due to the distance decrease. Normalization method is adopted for processing the maximum and minimum.

Let the relative increase in the amount, the actual distance, or the average distance be $x$. Let the amount of the increase normalized be $x_{s}$; see Table 2. Consider

$$
x_{s}=\frac{x-x_{\max }}{x_{\max }-x_{\min }} .
$$

Table 2 shows the relative increase of normalized actual distance and average distance.

Consolidated costs and optimized network efficiency are evaluated relative to the comprehensive increment in the amount of the actual distance of the average distance. If the relative increase of the actual distance is $a_{s}$ and the relative increase of the average distance is $a_{p}$, then the comprehensive increment is $a_{z}=a_{s}+a_{p}$. Assuming the cost and efficiency are of equal importance to the network, comprehensive increment of the actual distance is weighted equal to that of the average distance.

The candidate program is sorted from the superior to the inferior by the integrated incremental order in accordance with the policy set (Table 3). This paper selects the best of the three programs, namely, program 17 , program 8 , and program 14.

4.3. Optimization Programs. The selected three optimizations are original plans for the Guangzhou Railway Bureau with the following adjustments made. Option One: the cd-cs and hh-hy line are replaced by hh-yz and ld-hy line; Option Two: the hh-hy and cl-hy line are replaced by hh-yz and yz-sg line; Option Three: the cl-hy and hh-hy line are replaced by hh-yz and yz-gz line; see Figure 5.
TABLE 2: The actual distance and the relative increase in the average distance scales.

\begin{tabular}{lcc}
\hline $\begin{array}{l}\text { Program } \\
\text { number }\end{array}$ & $\begin{array}{c}\text { Relative increase of } \\
\text { actual distance }\end{array}$ & $\begin{array}{c}\text { Relative increase of } \\
\text { average distance }\end{array}$ \\
\hline 1 & 0.459839 & 0.399995 \\
2 & 0.793302 & 0.399995 \\
3 & 1 & 0.799992 \\
4 & 0.378309 & 0 \\
5 & 0.668881 & 0.399995 \\
6 & 0.06378 & 0.399995 \\
7 & 0.903425 & 0.799992 \\
8 & 0.331941 & 0 \\
9 & 0.223749 & 0.199996 \\
10 & 0.53596 & 0.599993 \\
11 & 0.689747 & 0 \\
12 & 0.3729 & 0 \\
13 & 0.76239 & 0.599993 \\
14 & 0.157675 & 0.199996 \\
15 & 0.49307 & 0.399995 \\
16 & 0.570349 & 0.199996 \\
17 & 0 & 0 \\
18 & 0.724523 & 1 \\
\hline
\end{tabular}

Using the network diagram, we can determine the adjacency matrix and the linear distance matrix to calculate initial relative entropy as 0.5375 . The microscopic optimization model can be calculated by the fault tolerance of convergence to the optimal 0.5392 and the visible microscopic optimization model can improve the network fault tolerance. To fulfill the optimal fault tolerance of the network, a large cluster is supposed to be selected according to the actual situation.

In an optimized network fault tolerance inspection, the average clustering coefficient and average distance are used 


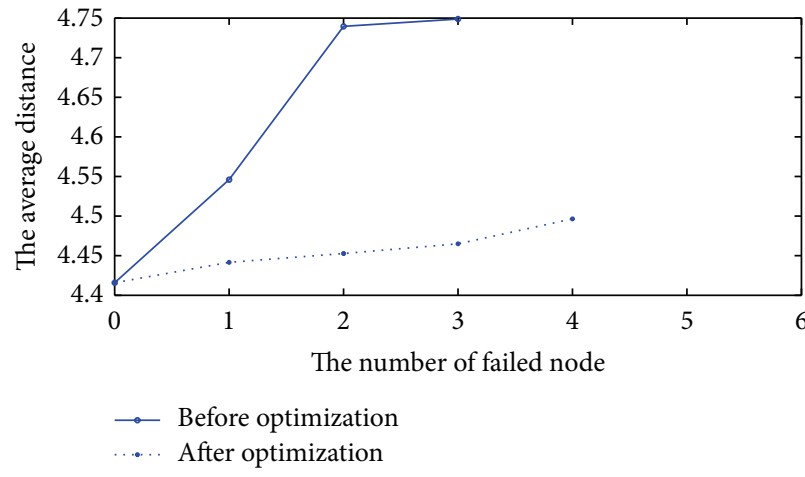

(a)

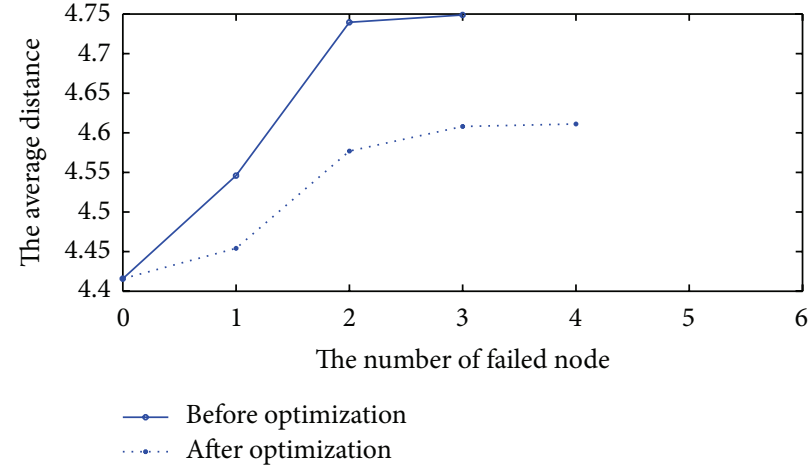

(b)

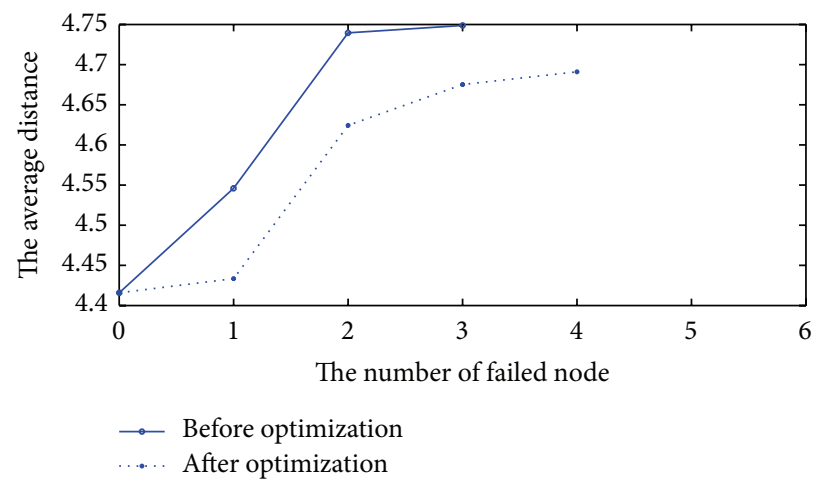

(c)

Figure 6: Micro fault tolerance before and after optimization.

TABLE 3: The order of integrated incremental.

\begin{tabular}{lccc}
\hline $\begin{array}{l}\text { Program } \\
\text { number }\end{array}$ & $\begin{array}{c}\text { Relative increase of } \\
\text { actual distance }\end{array}$ & $\begin{array}{c}\text { Relative increase of } \\
\text { average distance }\end{array}$ & $\begin{array}{c}\text { Integrated } \\
\text { incremental }\end{array}$ \\
\hline 17 & 0 & 0 & 0 \\
8 & 0.331941 & 0 & 0.331941 \\
14 & 0.157675 & 0.199996 & 0.357671 \\
12 & 0.3729 & 0 & 0.3729 \\
4 & 0.378309 & 0 & 0.378309 \\
9 & 0.223749 & 0.199996 & 0.423745 \\
6 & 0.06378 & 0.399995 & 0.463775 \\
11 & 0.689747 & 0 & 0.689747 \\
16 & 0.570349 & 0.199996 & 0.770345 \\
1 & 0.459839 & 0.399995 & 0.859834 \\
15 & 0.49307 & 0.399995 & 0.893065 \\
5 & 0.668881 & 0.399995 & 1.068876 \\
10 & 0.53596 & 0.599993 & 1.135953 \\
2 & 0.793302 & 0.399995 & 1.193297 \\
13 & 0.76239 & 0.599993 & 1.362383 \\
7 & 0.903425 & 0.799992 & 1.703417 \\
18 & 0.724523 & 1 & 1.724523 \\
3 & 1 & 0.799992 & 1.799992 \\
\hline
\end{tabular}

as indicators of fault tolerance. As Figure 5 illustrates, the micronetwork optimization model is an improved version compared with the original network. Under the same degree of random failures on average, the clustering coefficient decreases more slowly and the average distance increases more slowly as well and then collapses after a long duration. Figure 6 shows the specific performance as follows.

Average clustering coefficient:

(1) compared with the expansion of the scope of the attack, the rate of improved network's overall decline is slower than that of the original network;

(2) the improved network has a longer duration before collapse;

(3) with a small number of attack modes, the improved network has no significant change compared with the original network.

Average distance:

(1) as the scope of the attack expanded, the improved network one increases much slowly;

(2) with a small number of attack modes, the improved network has no significant change compared with the original one which increases drastically.

Therefore, it is concluded that the improved network has a better fault tolerance than the original one. 


\section{Conclusions}

In general, the primary aim of a railway network plan is to create a smooth traffic flow. With the consideration of random network breakdowns and failures, this paper maximizes the fault tolerance of railway networks and proposes the methods in enhancing the network from both macro and micro aspects. The study reveals that (1) the average node degree of "Twelfth Five-Year Plan" has greatly increased compared with China's 2008 network; (2) in order to maximize the fault tolerance, the average node degree in "Twelfth FiveYear Plan" should be raised; (3) Guangzhou Railway Bureau's railway network fault tolerance has been increased after the network's micro optimization.

This paper focuses on analyzing geographic railway network. Aligning with the power-law distribution, some studies argued that the traffic networks are scale-free networks requiring different optimization schemes. With relevant data, future researches and analysis about the fault tolerance will be conducted from the aspects of the traffic networks and transfer networks.

\section{Conflict of Interests}

The authors declare that there is no conflict of interests regarding the publication of this paper.

\section{Acknowledgment}

Project is supported by the Fundamental Research Funds for the Central Universities (Grant no. 2010QZZD021) and the Ministry of Railway Science and Technology Research Development Program (Grant no. 2012X012-A), China.

\section{References}

[1] R. Albert, H. Jeong, and A.-L. Barabasi, "Error and attack tolerance of complex networks," Nature, vol. 406, no. 6794, pp. 378-382, 2000.

[2] A. Broder, R. Kumar, F. Maghoul et al., "Graph structure in the web," Computer Networks, vol. 33, no. 1-6, pp. 309-320, 2000.

[3] R. Cohen, K. Erez, D. Ben-Avraham, and S. Havlin, "Resilience of the internet to random breakdowns," Physical Review Letters, vol. 85, no. 21, pp. 4626-4628, 2000.

[4] R. Cohen, K. Erez, D. Ben-Avraham, and S. Havlin, "Breakdown of the internet under intentional attack," Physical Review Letters, vol. 86, no. 16, pp. 3682-3685, 2001.

[5] T. Feng, H. Li, Z. Yuan, and J. Ma, "Analysis method of robustness for topology of Bernoulli Node model," Acta Electronica Sinica, vol. 20, no. 7, pp. 1673-1678, 2011 (Chinese).

[6] W. Wang, J. Liu, X. Jiang, and Y. Wang, "Topology properties on Chinese railway network," Journal of Beijing Jiaotong University, vol. 34, no. 3, pp. 148-152, 2010 (Chinese).

[7] H. Duan, Z. Li, and Y. Zhang, "Robustness analysis model of urban public transport network," Natural Science, vol. 38, no. 3, pp. 70-75, 2010 (Chinese).

[8] Y. Chen, J. Zhao, and H. Qi, "Robustness analysis for complex military network," Fire Control \& Command Control, vol. 35, no. 5, pp. 23-25, 2010 (Chinese).
[9] B. Shargel, H. Sayama, I. R. Epstein, and Y. Bar-Yam, "Optimization of robustness and connectivity in complex networks," Physical Review Letters, vol. 90, no. 6, Article ID 068701, 2003.

[10] G. Paul, T. Tanizawa, S. Havlin, and H. E. Stanley, "Optimization of robustness of complex networks," European Physical Journal B, vol. 38, no. 2, pp. 187-191, 2004.

[11] T. Tanizawa, G. Paul, R. Cohen, S. Havlin, and H. E. Stanley, "Optimization of network robustness to waves of targeted and random attacks," Physical Review E, vol. 71, no. 4, Article ID 047101, 2005.

[12] B. Wang, Evolution mechanism and some dynamical processes on complex network [Ph.D. thesis], Dalian University of Technology, 2006, (Chinese).

[13] C. He, Complex Network Properties of the Chinese Railway Network, Sun Yat-Sen University, Guangzhou, China, 2007 (Chinese).

[14] W. Zhao, H. S. He, Z. C. Lin, and K. Q. Yang, "Study of properties of Chinese railway passenger transport network," Acta Physica Sinica, vol. 55, no. 8, pp. 3906-3911, 2006 (Chinese). 


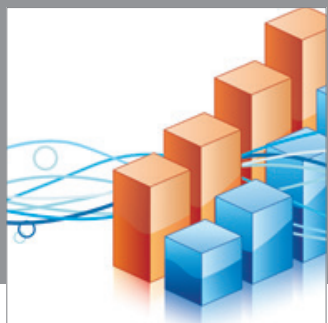

Advances in

Operations Research

mansans

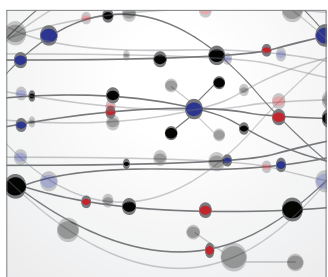

The Scientific World Journal
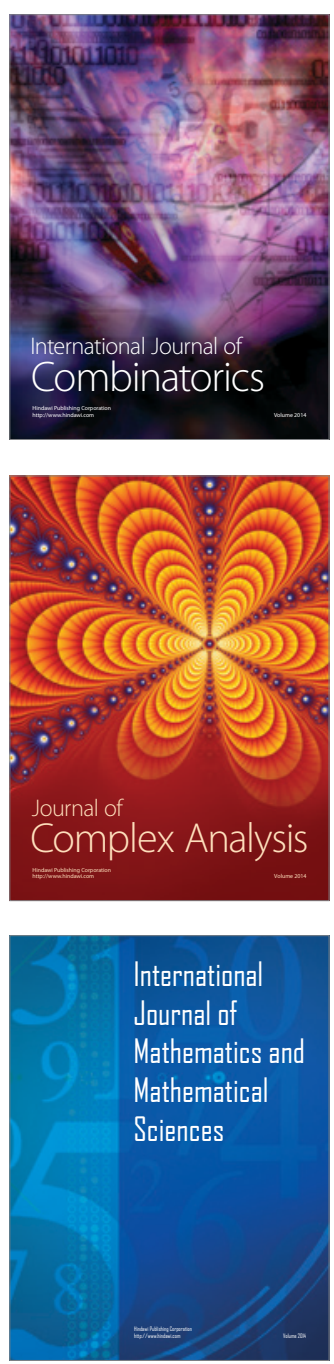
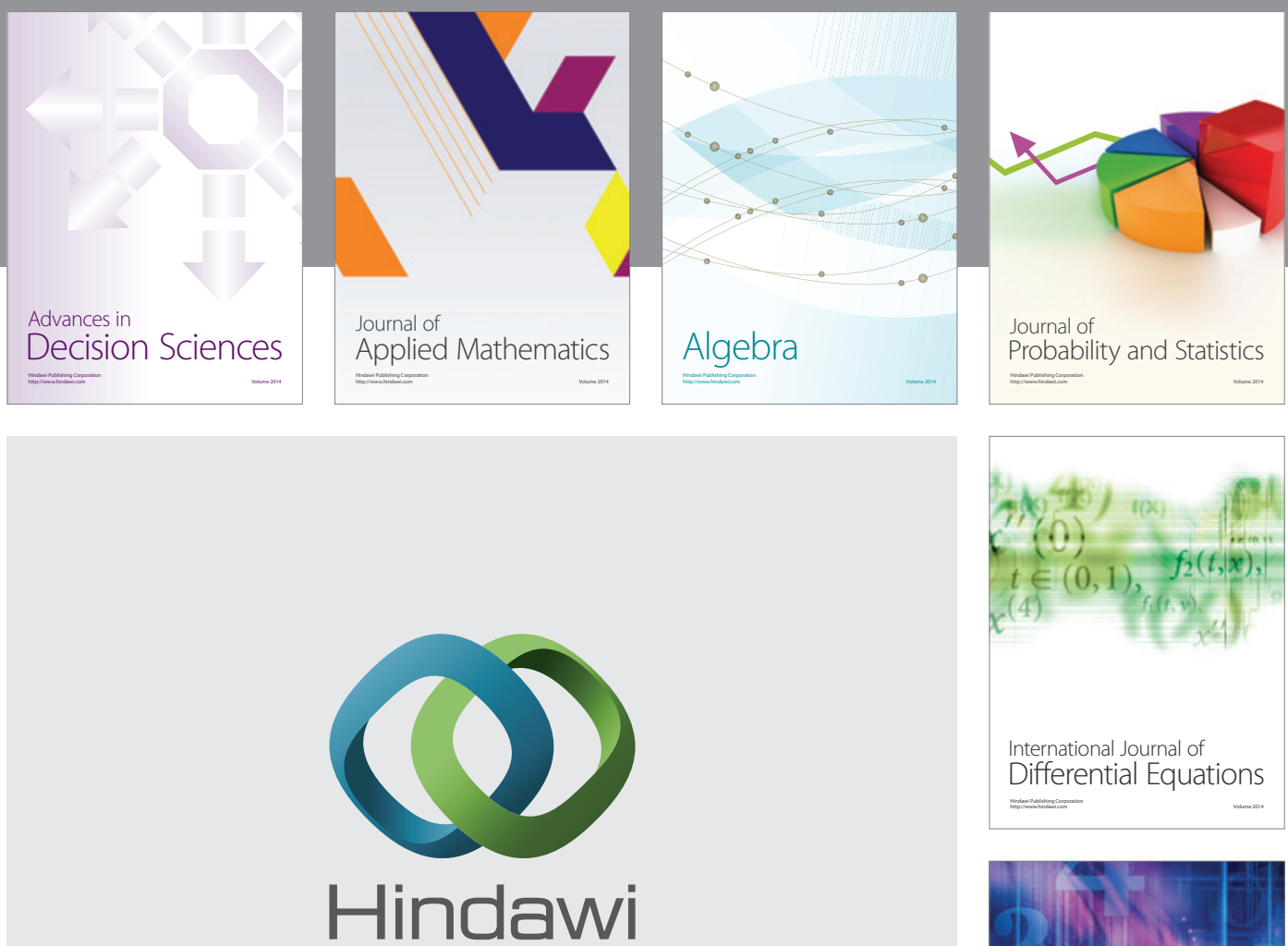

Submit your manuscripts at http://www.hindawi.com
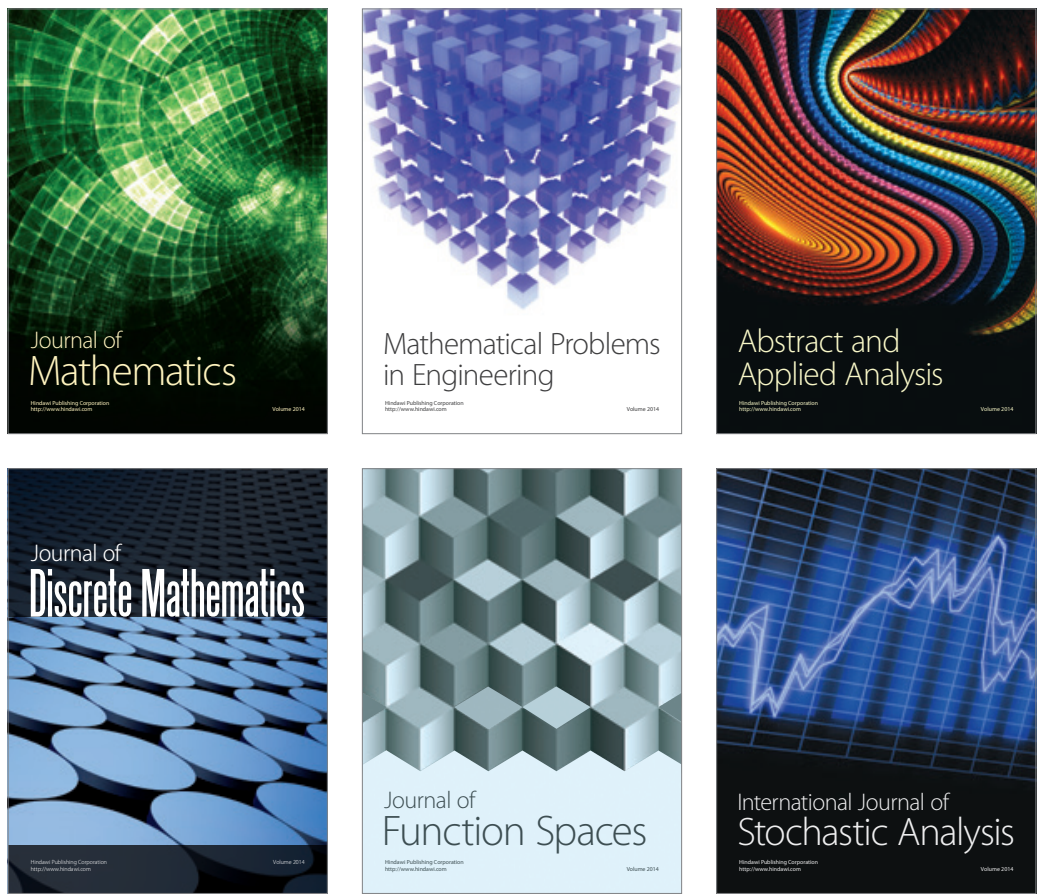

Journal of

Function Spaces

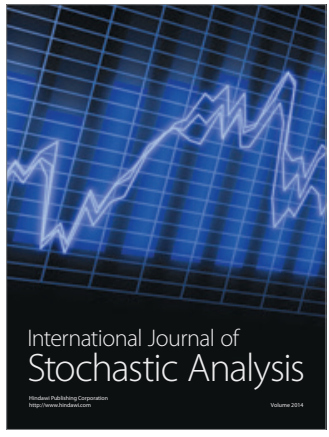

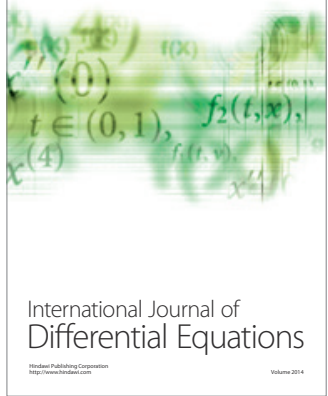
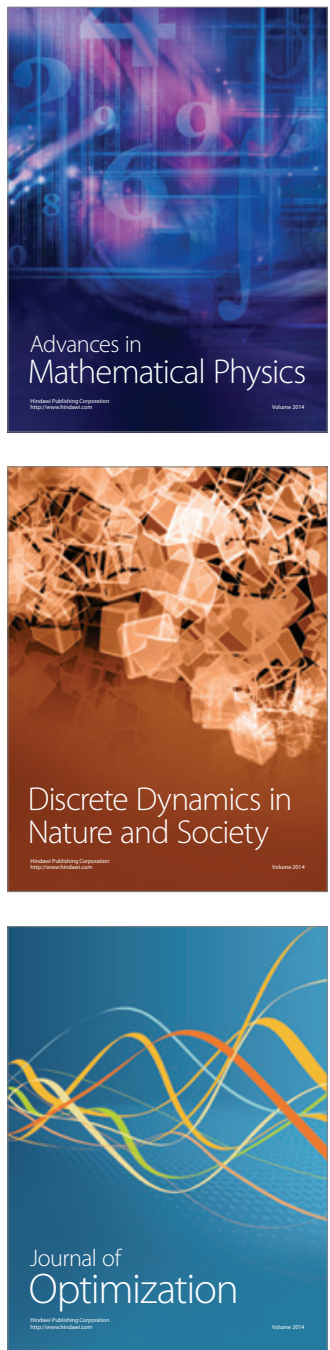